\title{
Beam-wall interaction in the CERN Proton Synchrotron for the LHC upgrade
}

\author{
M. Migliorati* and S. Persichelli \\ University of Rome "La Sapienza," Via A. Scarpa 14, 00161 Roma, Italy and CERN, Geneva 23, CH-1211, Switzerland \\ H. Damerau, S. Gilardoni, and S. Hancock \\ CERN, Geneva 23, CH-1211, Switzerland
}

L. Palumbo

University of Rome “La Sapienza," Via A. Scarpa 14, 00161 Roma, Italy

(Received 9 October 2012; published 20 March 2013)

\begin{abstract}
Coupling impedances and wakefields are fundamental quantities to characterize the electromagnetic interaction of a particle beam with the surrounding environment. In particular, collective effects, triggered by these self-induced fields, may play an important role in beam stability and machine performance. Within the framework of the LHC Injectors Upgrade project, since a significantly higher beam intensity is planned for the CERN Proton Synchrotron, wakefields are expected to increase their influence on the beam dynamics, and their evaluation is becoming important. In this paper we present the results of recent measurements of the longitudinal broadband coupling impedance by means of the incoherent quadrupole synchrotron frequency shift as a function of beam intensity. A detailed evaluation of the contribution of several machine installations to the total impedance budget is also presented and compared with the measurements.
\end{abstract}

DOI: 10.1103/PhysRevSTAB.16.031001

PACS numbers: 29.20.dk, 29.27.Bd, 41.85.Ew

\section{INTRODUCTION}

The longitudinal broadband impedance is an important parameter to characterize the electromagnetic coupling of a particle beam with the surrounding elements like kickers, radio-frequency (rf) cavities, diameter changes of the beam pipe, bellows, and many other installations close to the beam. The measured broadband impedance can be compared to the expected impedance obtained by evaluating the contributions of each relevant component. This may show whether all major contributing devices are well modeled and included. Additionally, in the framework of the LHC Injectors Upgrade project, important hardware changes are foreseen in the CERN Proton Synchrotron (PS), like new injection elements for an increased injection energy [1]. Based on the measurements presented in this paper, it will be possible to assess their impact on the broadband impedance.

The first estimations of the coupling impedance in the PS date back to the late 1970s. These measurements, based on longitudinal stability during debunching and on quadrupole beam transfer functions [2,3], were already indicating an impedance in the range of $Z(p) / p \simeq 25 \Omega$ [4]. A more refined measurement campaign of the broadband

\footnotetext{
*mauro.migliorati@uniroma1.it
}

Published by the American Physical Society under the terms of the Creative Commons Attribution 3.0 License. Further distribution of this work must maintain attribution to the author(s) and the published article's title, journal citation, and DOI. impedance [5], employing the same beam transfer function technique as used for the measurements presented in this paper, took place in the framework of the preparation of the PS as LHC injector. A broadband impedance of $Z(p) / p=$ $(21.7 \pm 5.1) \Omega$ was obtained.

With the improvement of the measurement techniques and a better control of the longitudinal beam parameters, notably the capability to produce bunches with constant longitudinal emittance over a wide intensity range, this paper presents the results of the latest measurement campaign.

The measurements have been performed by observing the quadrupolar beam transfer function and deducing the zero-amplitude synchrotron frequency as a function of bunch intensity. Similar longitudinal broadband impedance measurement campaigns in the CERN Super Proton Synchrotron (SPS) were regularly performed but by injecting a mismatched bunch and observing its quadrupole oscillations [6] instead of exciting the beam with noise.

In the following section we show the measurement setup and the results of the data analysis, from which an inductive broadband impedance has been derived. The coupling impedance has been used in a simulation code in Sec. III to reproduce the measurement results by means of what we can define as a "virtual measurement" and to confirm the validity of the method we used to obtain the machine impedance. Finally, in Sec. IV, we analyze the elements that mainly give an important contribution to the total coupling impedance, compare the results with those obtained from the measurements, and derive a longitudinal broadband impedance model. 


\section{MEASUREMENTS}

The measurements of the quadrupole synchrotron frequency were performed at a fixed momentum of $26 \mathrm{GeV} / \mathrm{c}$ [7] and with a single-harmonic rf system at $40 \mathrm{MHz}$. A single bunch with an intensity of up to $N_{p}=4.5 \times 10^{11}$ protons was injected from the PS Booster and accelerated in the PS on the 16th harmonic $(h=16)$ of the revolution frequency to a momentum of $26 \mathrm{GeV} / \mathrm{c}$. On the flat top, the bunch was first synchronized to a fixed revolution frequency of $f_{0}=476.82 \mathrm{kHz}$, allowing us to pulse a higher-harmonic rf cavity at $40 \mathrm{MHz}$, the 84th harmonic of the revolution frequency. About $150 \mathrm{~ms}$ before extraction the bunch was handed over from $h=16\left(f_{\mathrm{RF}}=7.629 \mathrm{MHz}\right)$ to $h=84$ $\left(f_{\mathrm{RF}}=40.052 \mathrm{MHz}\right)$. This rebucketing to the $40 \mathrm{MHz}$ rf system was completed $140 \mathrm{~ms}$ before extraction. Aside from a $5 \mathrm{~ms}$ time window for longitudinal emittance measurements, about $130 \mathrm{~ms}$ were left under stationary conditions to perform the beam transfer function (BTF) measurement. During that time, the bunch was held with a constant $40 \mathrm{MHz}$ rf voltage. No beam phase loop was active during the measurements. The length of the magnetic flat top and hence the measurement duration could not be stretched to avoid a too large heating of the main and auxiliary coils of the magnets, and averaging over many cycles was applied to improve the quality of the measurements.

\section{A. Measurement setup}

The spectrum of the incoherent quadrupole synchrotron frequency has been obtained by measurement of the longitudinal BTF with the setup sketched in Fig. 1. A bandwidth limited white noise (up to $2 \mathrm{kHz}$ ) was generated by the internal source of an Agilent 89410A vector network analyzer. This frequency range was chosen to fully cover the quadrupole synchrotron frequency and to achieve the best possible resolution within the $130 \mathrm{~ms}$ time window for the measurements. The noise signal was gated in order to only affect the beam during the well-defined duration of the measurement and was added as an amplitude modulation to the voltage program of the $40 \mathrm{MHz}$ cavity. Since the peak amplitude of the noise was independent of the voltage in the $40 \mathrm{MHz}$ rf cavity, the amplitude modulation index ranged from 0.07 to 0.13 . A copy of the noise signal used for amplitude modulation was directly fed to the reference channel of the vector network analyzer. Taking the noise signal to the reference channel directly from the source and not from the gap of the $40 \mathrm{MHz}$ cavity did not introduce a significant error, as the $3 \mathrm{~dB}$ bandwidth of the voltage control loop was measured to be of the order of $20 \mathrm{kHz}$. The delay between noise source and cavity gap does not introduce any significant phase shift in the relevant frequency range up to $2 \mathrm{kHz}$.

A wall-current monitor (WCM95) was used to pick up the longitudinal beam signal, followed by a peak detector with a time constant of several turns, but well below the quadrupole synchrotron oscillation period. The attenuation of the beam signal was chosen to get optimum signal amplitude to the peak detection circuit and special care was taken to avoid saturation. The peak-detected beam signal was then fed to the second channel of the vector network analyzer (see Fig. 1). The gating switch in front of the second input protects the measurement instrument from overvoltage, which may occur outside the measurement time window.

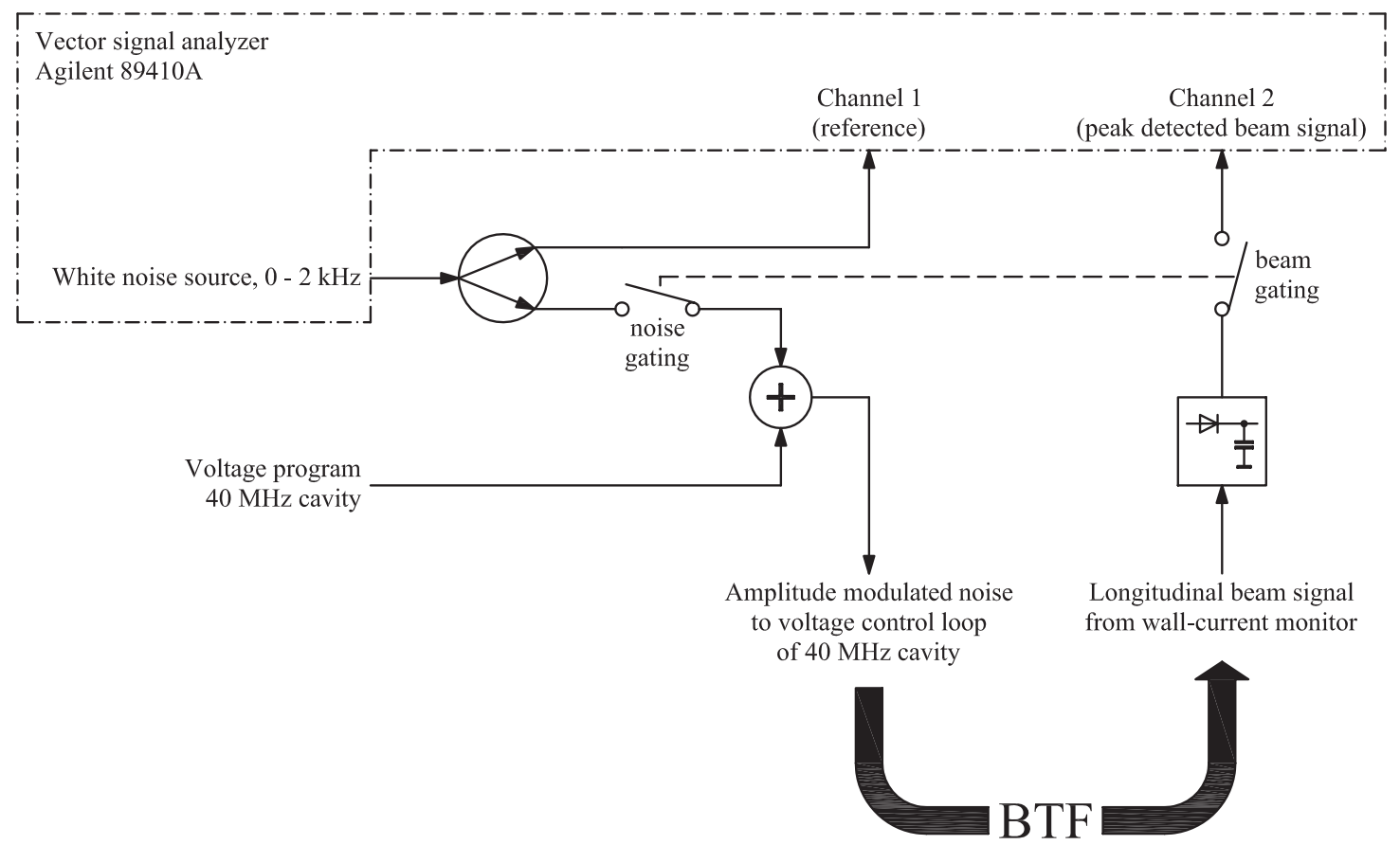

FIG. 1. Setup to measure the longitudinal quadrupole beam transfer function. 
Amplitude and phase of the quadrupole BTF are then determined by the analyzer by calculating the vectorial ratio of a peak-detected beam signal and noise excitation and subsequent averaging over many individual acceleration cycles. A typical averaged quadrupole synchrotron frequency spectrum is shown in Fig. 2.

The zero-amplitude quadrupole synchrotron frequency is given by the discontinuity of the phase curve following the $180^{\circ}$ phase advance $[8,9]$. Indeed, in a single-harmonic rf system, the incoherent synchrotron frequency decreases with the oscillation amplitude of the individual particle in the longitudinal phase space, due to the nonlinearity of the rf force. Particles in the center of the bunch thus have the highest, so-called zero-amplitude synchrotron frequency [10]. The bunch spectrum has a sharp cutoff at this frequency [11]. This cutoff forms the knee of the phase curve of the longitudinal beam transfer function. This welldefined point of the phase of the transfer function, when the beam response vanishes, is readily determined from the measurements. The small peak in both amplitude and phase at half that frequency is a direct observation of the synchrotron frequency due to residual dipole oscillations.

\section{B. Data analysis}

Two series of single-bunch measurements were made during two different machine development sessions. The momentum of $p_{0}=26 \mathrm{GeV} / \mathrm{c}$ was chosen to minimize the effects of space charge as will be shown in Sec. IV. We repeated the measurements at different rf peak voltages. Each measurement was taken by averaging over 16 acceleration cycles with approximately the same intensity and longitudinal emittance.

During the measurements the longitudinal bunch profile was recorded and the bunch length was obtained by fitting the profile both with a Gaussian and a parabolic line density distribution. Both models fit well the measured bunch profile, as shown in Fig. 3. The expected bunch deformation due to potential well distortion was too small to be observed directly, and all bunch profiles up to $N_{p}=$ $4.5 \times 10^{11}$ appear to be symmetric.

The results of the measurements, shown in Table II of the Appendix, have been used to obtain the low frequency longitudinal reactive impedance. The longitudinal equation of motion of a single particle in the presence of the self-induced wakefields is [12]

$\ddot{\tau}+\omega_{s 0}^{2} \tau=\frac{e N_{p} \omega_{s 0}^{2}}{V_{\mathrm{RF}} h \cos \phi_{s}} \sum_{p=-\infty}^{\infty} Z\left(p \omega_{0}\right) \sigma_{0}\left(p \omega_{0}\right) e^{i p \omega_{0} \tau}$,

with $\tau$ the position of the particle with respect to the synchronous one, $\omega_{s 0}$ the natural synchrotron frequency, $V_{\mathrm{RF}}$ the rf peak voltage, $h$ the harmonic number, $\phi_{s}$ the synchronous phase $\left(\cos \phi_{s}<0\right.$ above transition), $\omega_{0}$ the revolution frequency, $Z\left(p \omega_{0}\right)$ the longitudinal broadband coupling impedance, and $\sigma_{0}(\omega)$ the bunch spectrum of the stationary distribution.

In the absence of wakefields, the linear synchrotron motion has been assumed, and all the particles oscillate at the same frequency $\omega_{s 0}$ independently of their amplitude.
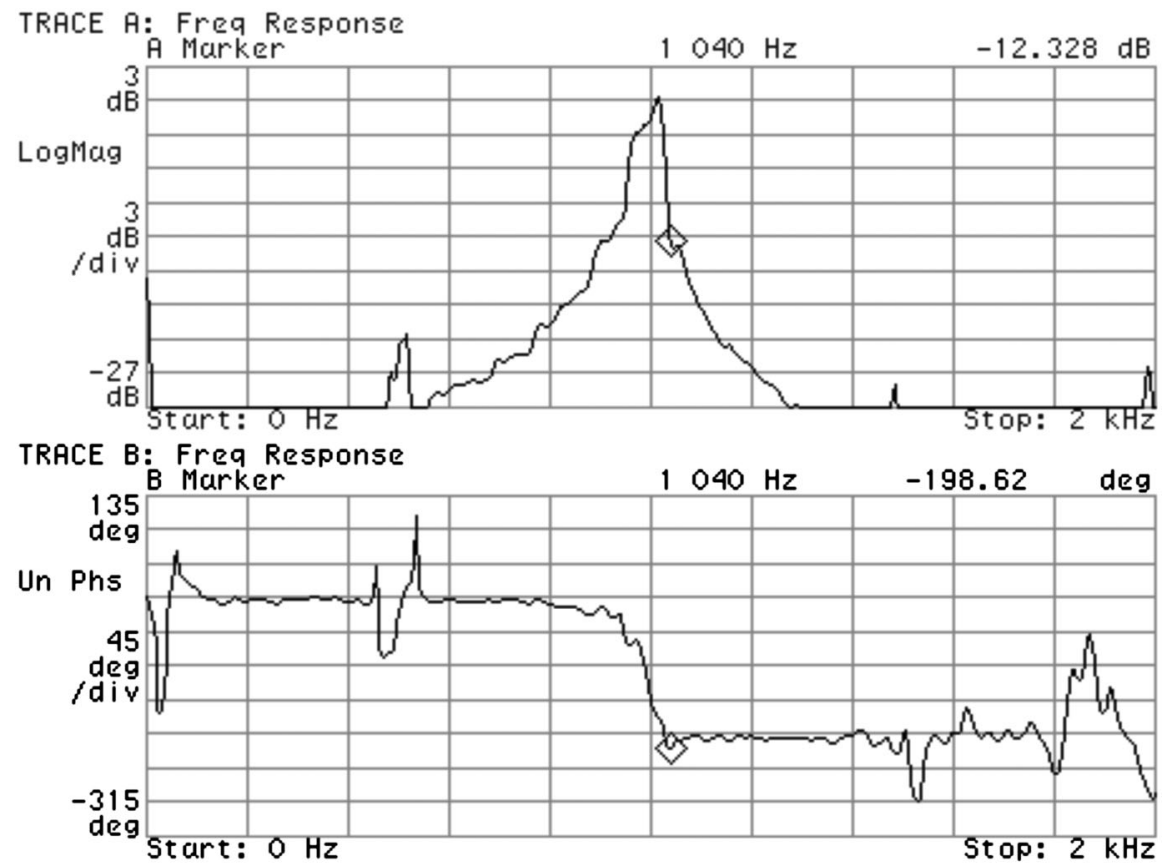

FIG. 2. Example screen shot of amplitude and phase of the quadrupole BTF for a bunch of about $9 \times 10^{10}$ protons kept by $95 \mathrm{kV}$ at $40 \mathrm{MHz}$. The diamonds indicate the zero-amplitude quadrupole synchrotron frequency. 


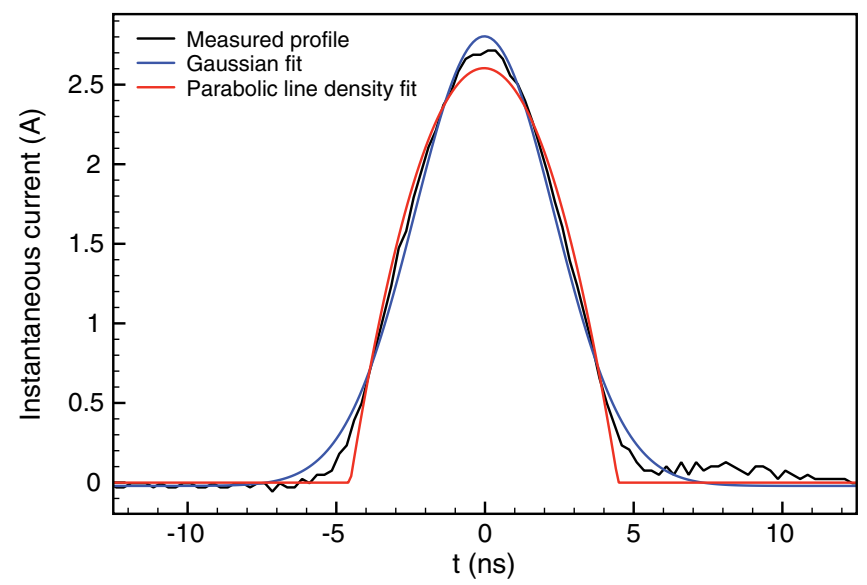

FIG. 3. Example of Gaussian and parabolic fit of the measured longitudinal bunch shape $\left(N_{p}=1.3 \times 10^{11}\right)$.

Stationary wakefields introduce several effects, including an incoherent quadrupole synchrotron frequency shift, which, for a Gaussian distribution, can be obtained from the expression $[12,13]$

$$
\begin{aligned}
\frac{f_{2 s}}{f_{s 0}} & =2+\frac{e N_{p}}{\sqrt{2 \pi} V_{\mathrm{RF}} h \cos \phi_{s} \omega_{0}^{2} \sigma_{G}^{3}} \frac{\operatorname{Im}[Z(p)]}{p} \\
& =2-\tilde{X}_{G} \frac{\operatorname{Im}[Z(p)]}{p},
\end{aligned}
$$

where $f_{2 s}$ is the quadrupole synchrotron frequency and $\sigma_{G}$ is the rms bunch length.

If we consider, instead, a parabolic line density and a pure inductive impedance, i.e., with constant $\operatorname{Im}[Z(p)] / p$, the quadrupole synchrotron frequency shift is derived from [12]

$$
\begin{aligned}
\frac{f_{2 s}}{f_{s 0}} & =2+\frac{12 e N_{p}}{V_{\mathrm{RF}} h \cos \phi_{s} \omega_{0}^{2} \tau_{b}^{3}} \frac{\operatorname{Im}[Z(p)]}{p} \\
& =2-\tilde{X}_{p} \frac{\operatorname{Im}[Z(p)]}{p}
\end{aligned}
$$

with $\tau_{b}$ the total bunch length.

The two expressions come from the same initial equation of motion, Eq. (1), but derived with different methods. For the Gaussian distribution, a linearization of the coherent force by taking only the first order expansion of the exponential term $e^{i p \omega_{0} \tau}$ has been used, while for the parabolic line density case, the infinite summation on the righthand side of Eq. (1) has been expressed in a closed form and a coherent force linear in $\tau$ has been obtained without any approximation.

If we compare Eq. (2) with Eq. (3), by considering $\tau_{b} \simeq 4 \sigma_{G}$, we observe that the parabolic line density predicts a frequency shift about a half of that obtained with the first order expansion and a Gaussian distribution for the same impedance. In other words, the parabolic model tends to provide twice larger impedance than the Gaussian model for the same measured quadrupole synchrotron frequency shift. A quadrupole frequency shift derived with the same method that led to Eq. (2) but by using a parabolic amplitude distribution has already been presented in [12], where the same factor 2 difference with the parabolic line density distribution has been evidenced. This difference is then not the result of the longitudinal distribution function but rather of the truncation in the expansion of the exponential term of Eq. (1).

By using the results of the measurements, the normalized incoherent quadrupole synchrotron frequency as a function of $\tilde{X}$ can be plotted for both of the models, as shown in Fig. 4. The error bars have been obtained by using the uncertainty propagation from the measured data. The slope of the linear regression, obtained with the method of least squares, gives directly the broadband longitudinal reactive impedance of the machine, which is $\operatorname{Im}[Z(p)] / p=(18.4 \pm$ 2.2) $\Omega$ for the parabolic line density distribution and $\operatorname{Im}[Z(p)] / p=(9.7 \pm 1.3) \Omega$ for the Gaussian distribution.

The factor of 2 in the values of $\operatorname{Im}[Z(p)] / p$, obtained by using the two distribution models, is confirmed by the results of simulations, which indicate that the parabolic line density distribution is more suited to the PS case, as we will show in the next section, where the same analysis technique used for the measurements has been applied to simulations. Moreover we observe that an impedance of $\operatorname{Im}[Z(p)] / p=(18.4 \pm 2.2) \Omega$ is very close to the results obtained in previous measurements [5,14].

To conclude the data analysis, we observe that, during our measurements, most probably the PS machine was operated below the microwave instability threshold. In fact, by applying the Boussard criterion [15], the singlebunch intensity threshold is

$$
N_{p}=\frac{(2 \pi)^{3 / 2} \eta\left(E_{0} / e\right) \sigma_{G} \sigma_{p}^{2}}{e|Z(p) / p|},
$$

with $\eta$ the slippage factor and $\sigma_{p}$ the rms relative momentum spread. With the typical beam parameters of the

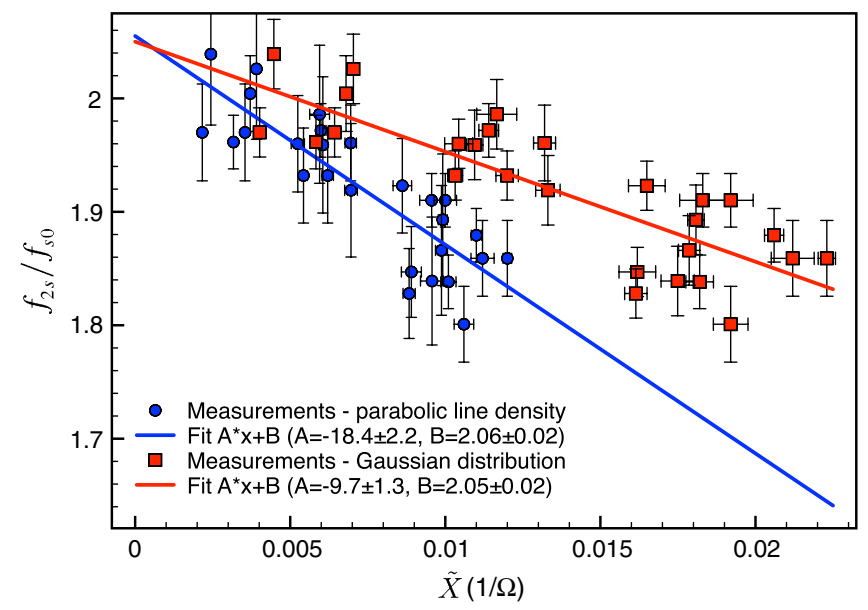

FIG. 4. Quadrupole frequency shift and linear fit with Gaussian and parabolic line density distributions. 
measurements, that is $\eta=2.53 \times 10^{-2}, \sigma_{G}=2.3 \mathrm{~ns}$, and $\sigma_{p}=3.6 \times 10^{-4}$, we get $N_{p} \simeq 10^{12}$, which is more than a factor of 2 above the maximum intensity used for the measurements. Moreover, previous observations of bunch profiles with a sampling rate of $20 \mathrm{Gs} / \mathrm{s}$ during the first turn in the SPS did not show evidence of a microwave instability in the PS [16].

\section{SIMULATIONS AND ANALYTICAL RESULTS}

Simulations of longitudinal beam dynamics, taking into account the collective effects of wakefields, are a powerful tool to better understand the beam behavior. We have therefore performed a series of simulations by using a tracking code initially developed to study the longitudinal beam dynamics in the electron storage ring DAФNE at LNF-INFN $[17,18]$ and recently adapted to the beam parameters of the CERN PS. For the simulations we have used $10^{5}$ macroparticles.

In order to check which distribution model is most appropriate in our case to evaluate $\operatorname{Im}[Z(p)] / p$, we have performed some "virtual measurements" by means of the simulation code. Indeed, we can track the synchrotron oscillations of each macroparticle and, by means of the fast Fourier transform, obtain the corresponding frequency spectra. By including the collective effects due to the wakefields, an incoherent quadrupole frequency shift as a function of beam intensity can be extracted. In Fig. 5 an example of the fast Fourier transform phase of the incoherent frequency spectrum around $\omega=2 \omega_{s 0}$ is shown at different bunch intensities with a pure inductive impedance of $\operatorname{Im}[Z(p)] / p=18.4 \Omega$, and it can be compared to the phase of the measured beam signal shown in Fig. 2. A quadrupole synchrotron frequency decreasing with bunch intensity is clearly visible in the zoomed portion.

By performing the same analysis as with the measured data, we obtain the plots of Fig. 6, with the Gaussian

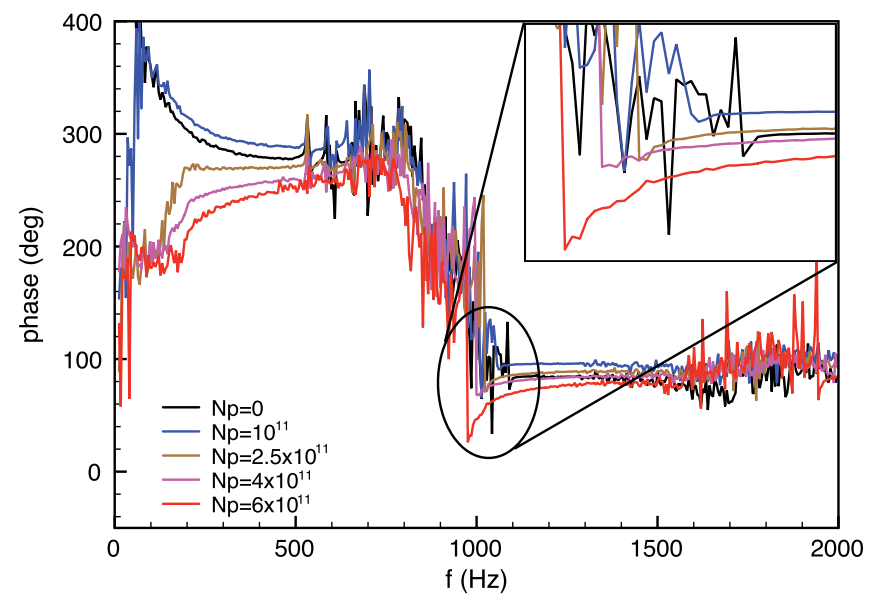

FIG. 5. Phase of the incoherent frequency spectrum at different bunch intensities obtained with the simulation code with an imaginary impedance of $\operatorname{Im}[Z(p)] / p=18.4 \Omega$.

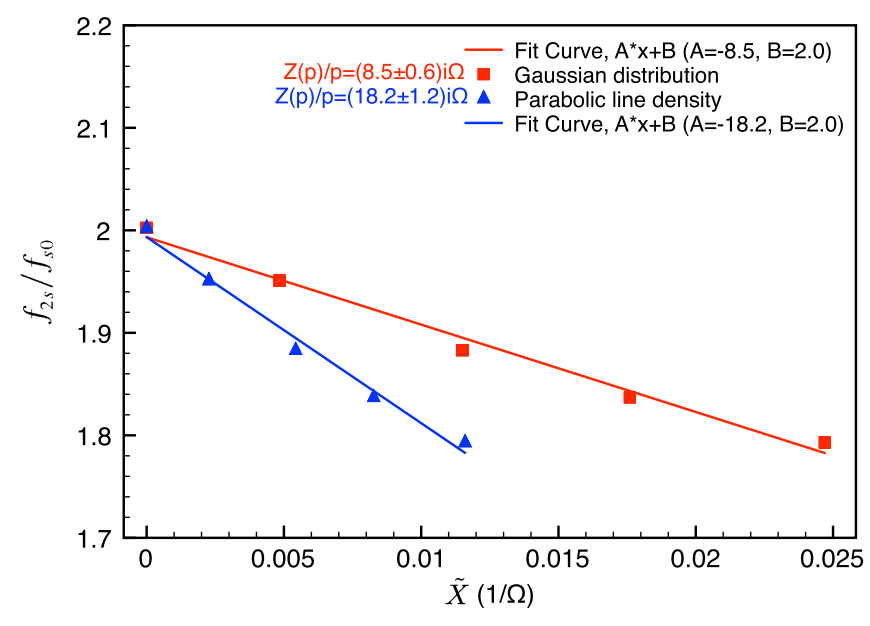

FIG. 6. Quadrupole frequency shift and linear fit with Gaussian distribution and parabolic line density by using the simulation results.

distribution giving a reactive impedance $\operatorname{Im}[Z(p)] / p=$ $(8.5 \pm 0.6) \Omega$, which is about half the value of our input impedance, while with the parabolic line density and Eq. (3) we obtained $\operatorname{Im}[Z(p)] / p=(18.2 \pm 1.2) \Omega$, very close to the measured value. From these results we can conclude that, for the PS parameters, the more appropriate expression for the analysis of our measurements is given by Eq. (3).

In Fig. 7 we show the comparison of the quadrupole synchrotron frequency shift of the simulations and of the measurements for the parabolic line density distribution. The agreement is very good, indicating that, with a simple model of inductive broadband impedance, we are able to reproduce the measurement results. There is a small displacement between the two fit curves, the intercept of the measurements being a bit too high (it should not be greater than 2). However, this overestimation is within the measurement uncertainty of $\pm 3 \sigma$.

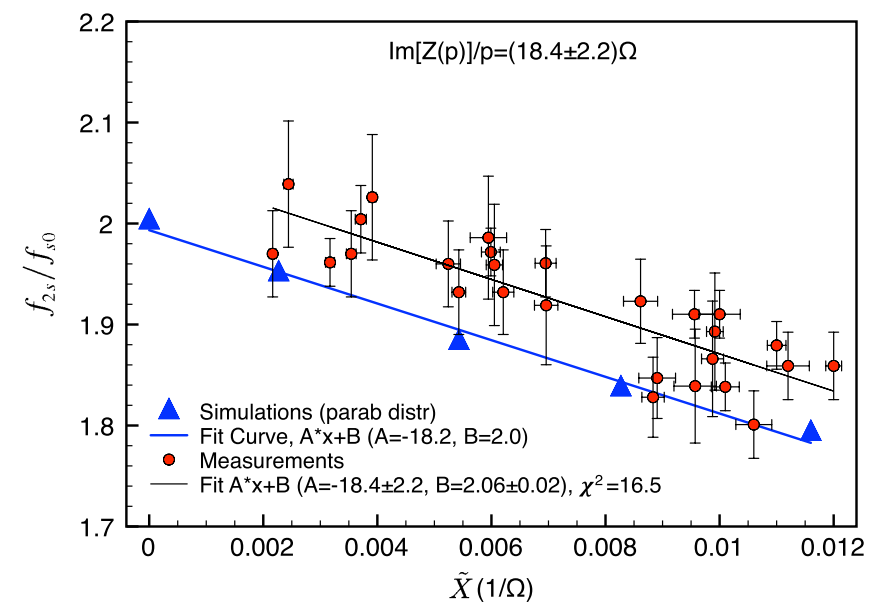

FIG. 7. Comparison between the incoherent quadrupole frequency shift obtained with the simulations and the results of the two sets of measurements. 


\section{THE PS BROADBAND IMPEDANCE MODEL}

In this section we evaluate the broadband longitudinal coupling impedance we expect in the PS by taking into account important contributions of the relevant machine installations, and we compare the results with the measured impedance.

The choice of performing the measurements at the flattop energy of about $26 \mathrm{GeV}$ has been made to reduce the contribution of the direct space charge (capacitive above transition) to the broadband impedance. The space charge impedance due to the nonrelativistic velocity of the charges in a circular pipe of radius $b$ can be written in the form [19-22]

$$
\frac{Z(p)}{p}=-i \frac{Z_{0}}{\beta \gamma^{2}} g_{l},
$$

with $Z_{0}=377 \Omega$ the impedance of the free space, $\gamma$ the relativistic Lorentz factor, and $g_{l}$ a geometric factor depending on the transverse bunch distribution. In particular, for a round beam with a uniform transverse distribution of radius $a$, we obtain the widely used expression

$$
g_{l}=\ln \frac{b}{a}+\frac{1}{2}
$$

In the case of an elliptic vacuum chamber, it is possible to substitute $b$ with an equivalent radius related to elliptic functions [23], which, for the PS case (semiaxes $35 \times$ $73 \mathrm{~mm}$ ), is $b=43 \mathrm{~mm}$. The absolute value of the imaginary part of the space charge impedance is shown in Fig. 8 as a function of $\gamma$. The contribution to the total broadband impedance at the energy of our interest is $\operatorname{Im}[Z(p)] / p=-1.9 i \Omega$.

A very important contribution to the total machine impedance is given by the several ferrite loaded kickers of the PS [24]. The longitudinal impedance for these kickers has been evaluated by using the field matching

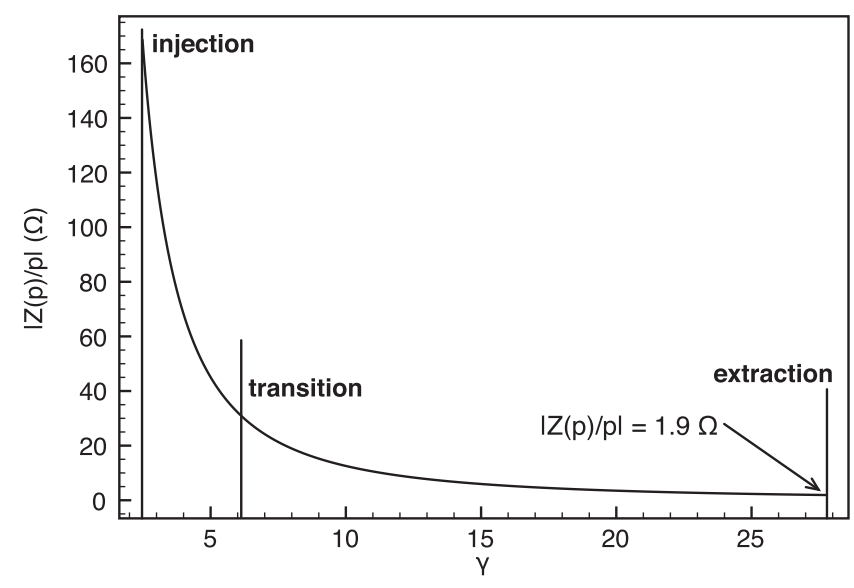

FIG. 8. Absolute value of the imaginary part of the space charge impedance for the PS as a function of the relativistic factor $\gamma$.

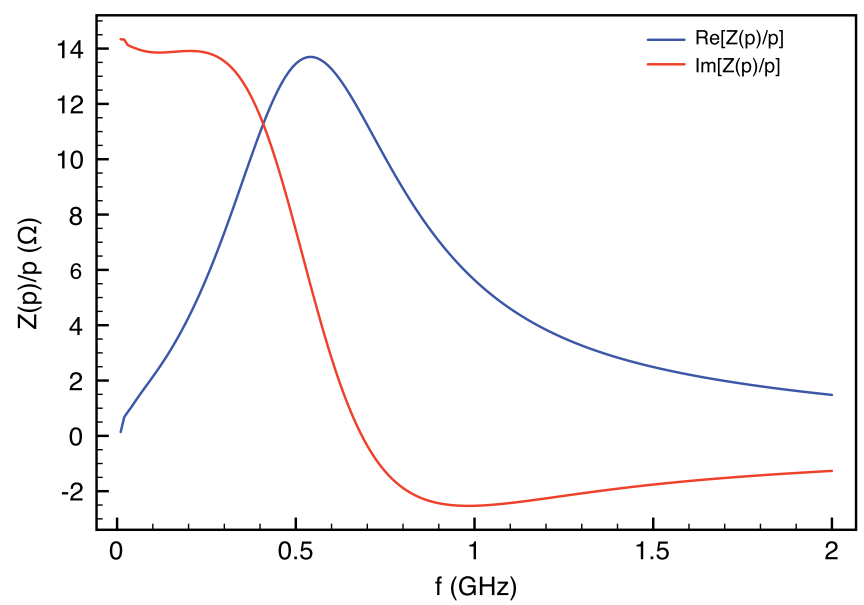

FIG. 9. PS kickers longitudinal impedance.

technique [25], which was shown to be in good agreement with measurements [26] and Computer Simulation Technology Particle Studio (CST-PS) [27] simulations [28]. The total longitudinal impedance of all the kickers is shown in Fig. 9.

Also the connection regions between the beam pipes and the vacuum pumps give an important contribution to the geometrical impedance, especially since about 100 of these pump ports are present. Indeed the connection is not a simple hole, and there is no rf shielding in the port of the pumps. In Fig. 10 we show a sketch of two of these structures.

The length of the cylindrical pipe connecting the beam pipe to the vacuum pump does not affect, at first order, the coupling impedance. Simulations have been performed with CST-PS and the results are shown in Fig. 11. The impedance is only inductive and, for a single pump, its value is $Z(p) / p=2.8 \times 10^{-2} i \Omega$.

We have also evaluated the impedance due to the resistive wall. For a circular pipe of radius $b$ with high conductivity $\sigma_{c}$, such that $c^{2} /\left(\omega^{2} b\right)$ and $b$ are much bigger than the skin depth $\delta$, the coupling impedance is given by

$$
\frac{Z(p)}{p}=\frac{Z_{0} \delta}{2 b}[1+i \cdot \operatorname{sgn}(\omega)]
$$

In the case of an elliptic vacuum chamber, as in the PS, $b$ represents the minor semiaxis, and the impedance has to be multiplied by a form factor that depends on the ellipticity

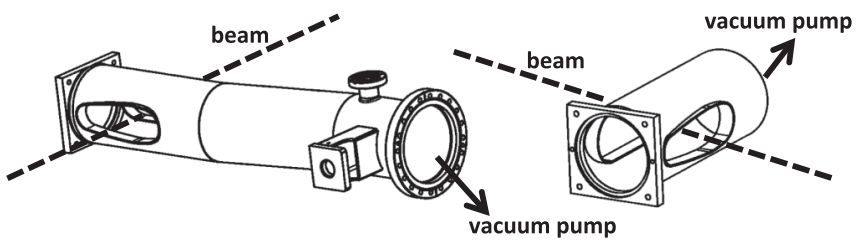

FIG. 10. Sketch of two vacuum pump connections with the beam pipe. 


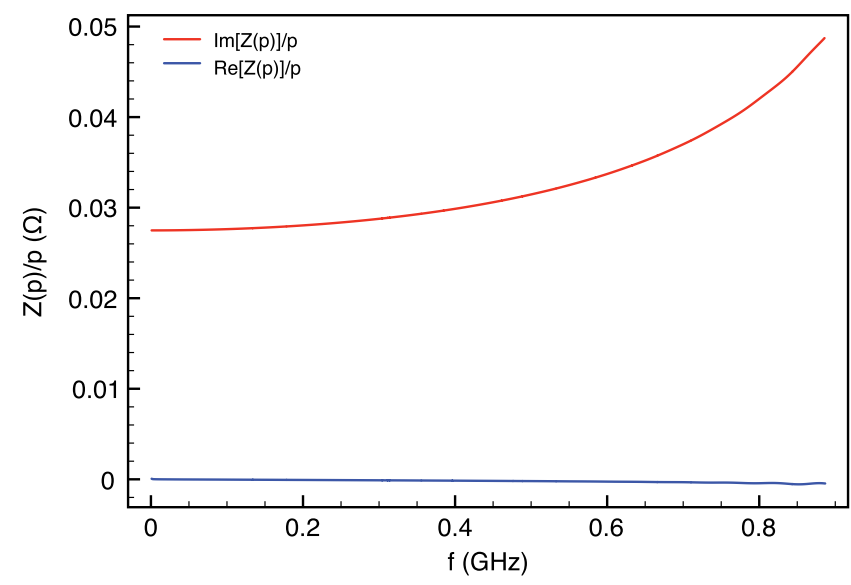

FIG. 11. Impedance of a vacuum pump connection to the beam pipe, obtained with CST-PS.

of the beam pipe [29-31]. For the PS vacuum chamber, this form factor is about 0.96. The skin depth depends on the pipe material, which, in our case, is stainless steel 316 LN (about $70 \%$ of the machine, with conductivity $\sigma_{c}=$ $1.3 \times 10^{6} \mathrm{~S} / \mathrm{m}$ and thickness of $2 \mathrm{~mm}$ ) and Inconel X750 alloy (about $20 \%$ of the machine, with conductivity $\sigma_{c}=$ $8.3 \times 10^{5} \mathrm{~S} / \mathrm{m}$ and thickness of $1.5 \mathrm{~mm}$ ) [32]. The two conductivities give skin depths, respectively, of $\delta=$ $0.6 \mathrm{~mm}$ and $\delta=0.8 \mathrm{~mm}$ at the PS revolution frequency, both smaller than the material thickness. The impedance contribution due the resistive wall is shown in Fig. 12 for both materials as a function of frequency. The impedance at the revolution frequency [33] is $Z(p) / p=2.2(1+i) \Omega$ for the stainless steel $316 \mathrm{LN}$ and $Z(p) / p=0.8(1+i) \Omega$ for the Inconel X750 alloy, while at the bunch spectrum cutoff [34] it is $Z(p) / p=0.07(1+i) \Omega$ for the stainless steel $316 \mathrm{LN}$ and $Z(p) / p=0.02(1+i) \Omega$ for the Inconel X750 alloy.

Concerning the rf cavities, we have taken into account the effects of the resonant modes due to the $10 \mathrm{MHz}$ cavities (for a total of 10 cavities), one $20 \mathrm{MHz}$ cavity, short

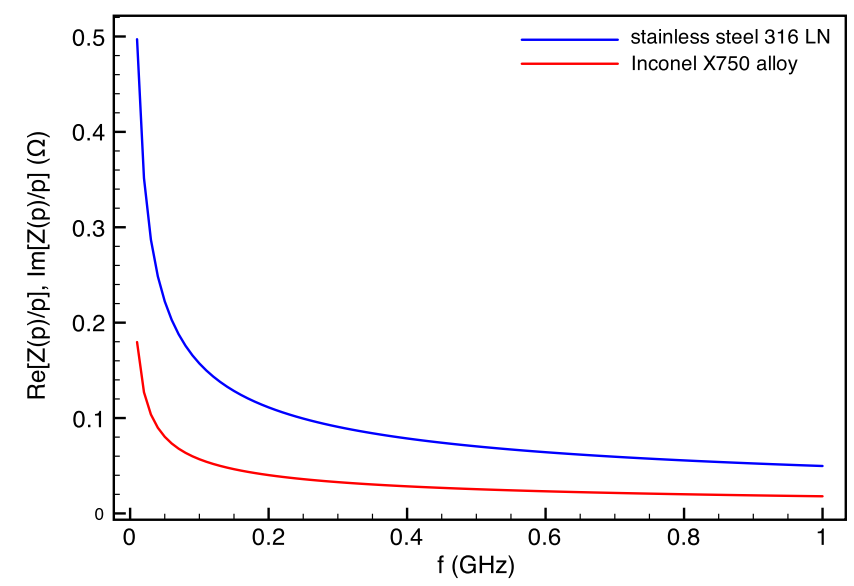

FIG. 12. Resistive wall impedance for the PS vacuum chamber.

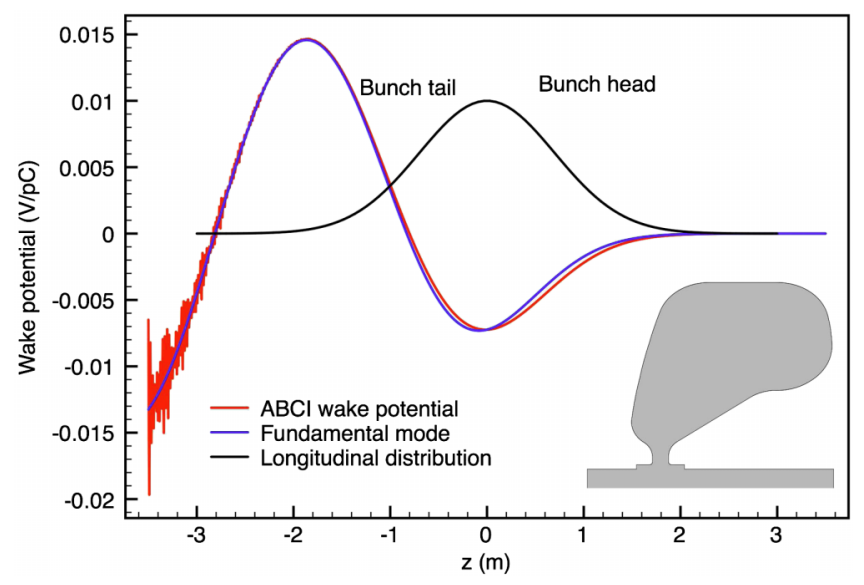

FIG. 13. $\quad 80 \mathrm{MHz}$ cavity shape and wake potential of a $2.3 \mathrm{~ns}$ Gaussian bunch obtained by ABCI and by accounting only for the contribution of the fundamental mode.

circuited during our measurements, one $40 \mathrm{MHz}$, and two $80 \mathrm{MHz}$ cavities. All of them can be approximated by single resonance oscillators, as their gaps are short compared to the bunch length and their first higher order modes are well above the fundamental resonance. Their contribution to the total machine broadband impedance is mainly resistive. As an example, in Fig. 13, we show the shape of the $80 \mathrm{MHz}$ cavity, and the wake potential of a $\sigma_{G}=2.3$ ns Gaussian bunch, obtained with the numerical code ABCI (Azimuthal Beam Cavity Interaction) [35] (red line), compared to the wake potential obtained by accounting only for the contribution of the fundamental mode (blue line) [36].

In addition to the pumping ports, an important source of geometrical impedance is due to the numerous step transitions of the PS vacuum chamber. In Fig. 14 we present the vertical apertures (horizontal and vertical) along the machine, showing many discontinuities [37].

A simplified expression for the low frequency impedance of a step transition in a circular beam pipe can be obtained by solving two quasistatic problems, electrostatic and magnetostatic [38], and it can be written as

$$
\frac{Z(p)}{p}=i \frac{\omega_{0} Z_{0} h^{2}}{4 \pi^{2} b c}\left(2 \ln \frac{2 \pi b}{h}+1\right)
$$

with $h$ the height of the step. By considering a circular pipe from 4 to $8 \mathrm{~cm}$, we obtain $Z(p) / p=1.8 \times 10^{-2} i \Omega$. The above expression is, however, approximate for the PS where the beam pipe is elliptic and, as can be seen in Fig. 14, the steps are not the same in the horizontal and vertical planes. With CST-PS, a simulation of a step out from an elliptic pipe of $35 \times 73 \mathrm{~mm}$ to a circular one of $73 \times 73 \mathrm{~mm}$ gives an imaginary impedance of $Z(p) / p=$ $8 \times 10^{-3} i \Omega$, about half of the value predicted by Eq. (8), as it should be, due to the fact that the step in the horizontal plane is zero in this case.

Other methods to calculate the impedance of a step in a circular pipe take into account the propagating modes of 


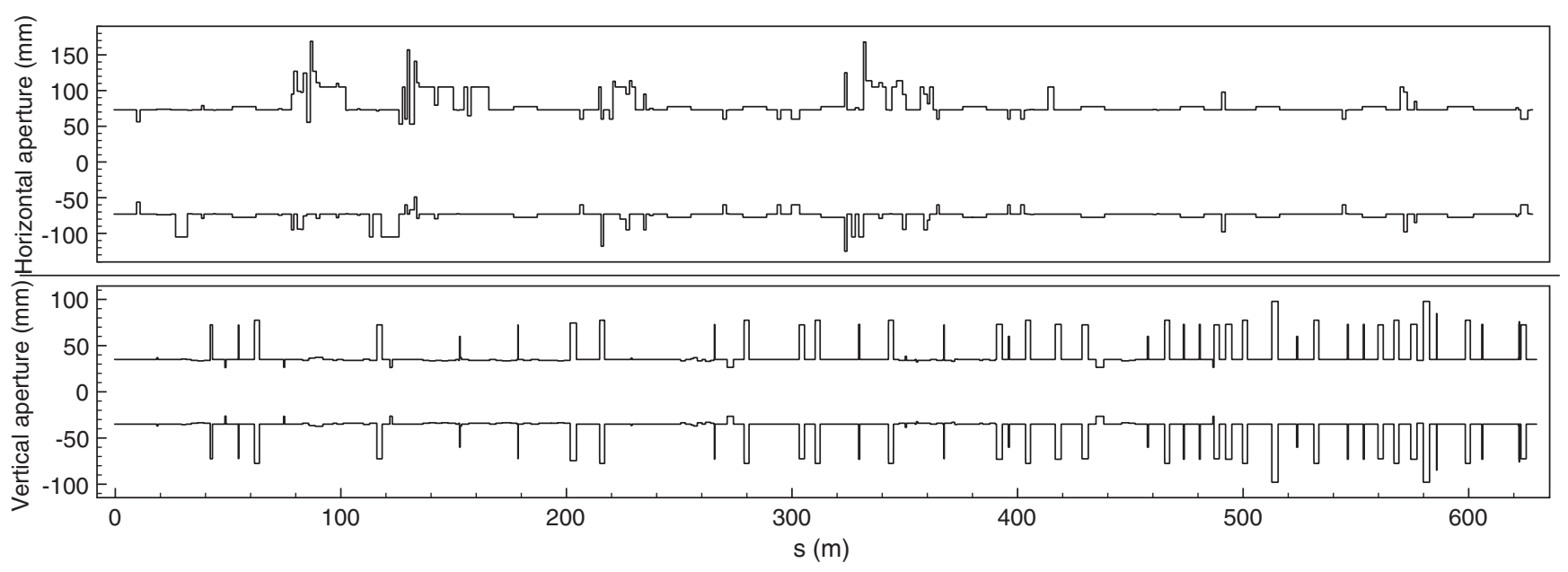

FIG. 14. Horizontal and vertical physical apertures of the PS vacuum chamber.

two semi-infinite waveguides, into which the vacuum chamber can be divided, and use boundary conditions at the step transition $[39,40]$. In all cases, the resulting impedance for our parameters is purely inductive giving, for the couple of step out/in configurations, a value of about $\operatorname{Im}[Z(p)] / p=1.6 \times 10^{-2} i \Omega$.

Another important inductive element is represented by the bellows. The impedance at low frequency can be obtained from that of a short pillbox with a width $w$ much lower that the height $h$, and it is given by [33]

$$
\frac{Z(p)}{p}=i \frac{n_{c} \omega_{0} Z_{0}}{2 \pi b c}\left(w h-\frac{w^{2}}{2 \pi}\right)
$$

with $n_{c}$ the number of corrugations per bellow.

For the PS case we have assumed $w=3 \mathrm{~mm}, h=$ $14 \mathrm{~mm}$, eight corrugations per bellow, and two bellows for each of the 100 dipoles. The total impedance, by considering a circular pipe cross section, is about $Z(p) / p=1.1 i \Omega$. However, the chamber of the bellows is not circular, so we expect a bit lower value. Indeed,
CST-PS simulations, the results of which are shown in Fig. 15, give a total inductive impedance of $Z(p) / p=$ $0.85 i \Omega$, anyway very close to the one evaluated by using the circular cross section formula.

In Table I we summarize the contributions to the machine impedance due to all the installations that have been evaluated.

In order to compare the study of this section with the measured broadband reactive impedance, since for some elements $Z(p) / p$ is not constant but a function of frequency, we have obtained the wake potential of a Gaussian distribution with $\sigma_{G}=2.3 \mathrm{~ns}$ in both cases. The choice of the bunch length is motivated by the fact that this is one of the shortest bunches we used in the measurements. Figure 16 shows that measured and calculated wake potentials are very close to each other, indicating that an inductive impedance is a fairly good model for studying the longitudinal single-bunch beam dynamics of the PS.

Instead of a purely inductive impedance, we could also use a broadband resonator model with an impedance of the kind
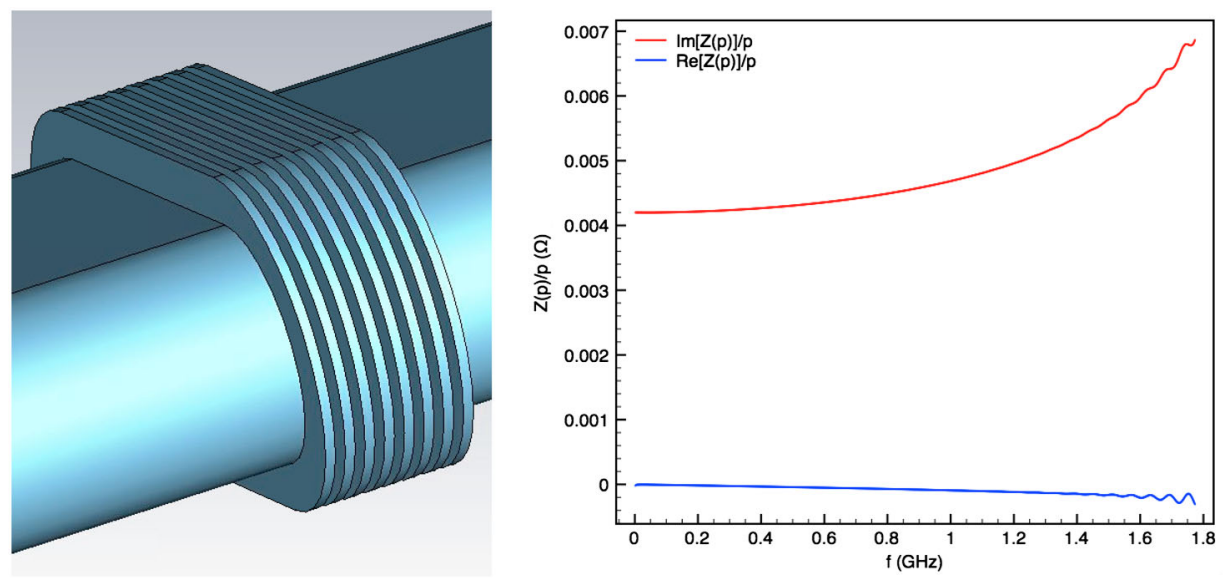

FIG. 15. Impedance of a bellow obtained with CST-PS simulations. 
TABLE I. Impedance contribution of important machine elements evaluated at the bunch spectrum cutoff. For the rf cavities the fundamental mode parameters are reported, including fast $\mathrm{rf}$ feedback. As the $200 \mathrm{MHz}$ cavities used for longitudinal blowup have no relevant contribution, they are not included in the table.

\begin{tabular}{lc}
\hline \hline Machine element & $Z(p) / p$ at $\omega=1 / \sigma_{G}$ \\
\hline Space charge & $-1.9 i \Omega$ \\
Kickers & $(1.6+13.8 i) \Omega$ \\
Pumping ports & $2.8 i \Omega$ \\
Resistive wall & $0.09(1+i) \Omega$ \\
Steps & $0.96 i \Omega$ \\
Bellows & $0.85 i \Omega$ \\
\hline \hline
\end{tabular}

\begin{tabular}{lrccc}
\hline \hline$f(\mathrm{MHz})$ & $Q$ & $R / Q(\Omega)$ & Number of cavities & Comment \\
\hline 7.6 & 5 & 30 & $10(+1$ spare $)$ & spare short circuited \\
20 & 4.6 & 43.5 & $1(+1$ spare $)$ & both short circuited \\
40 & 70 & 33 & $1(+1$ spare $)$ & a \\
80 & 100 & 56 & $2(+1$ spare $)$ & a \\
\hline \hline
\end{tabular}

${ }^{\mathrm{a}}$ Spare shielded from beam by mechanically closed gap.

$$
Z(\omega)=\frac{R_{s}}{1+i Q\left(\frac{\omega}{\omega_{r}}-\frac{\omega_{r}}{\omega}\right)}
$$

with $R_{s}$ the shunt impedance, $Q$ the quality factor, and $\omega_{r}$ the resonant frequency. At low frequency $\omega \ll \omega_{r}$, this can be approximated with

$$
Z(\omega \rightarrow 0) \simeq i \frac{R_{s} \omega}{Q \omega_{r}}
$$

If we consider a quality factor equal to 1 [41] and a resonant frequency equal to the frequency cutoff of the elliptic beam pipe [42], which in the PS is $2.54 \mathrm{GHz}$, with $Z(p) / p=18.4 i \Omega$, we get $R_{s} \simeq 98 k \Omega$. The wake potential of this broadband resonator impedance for a Gaussian

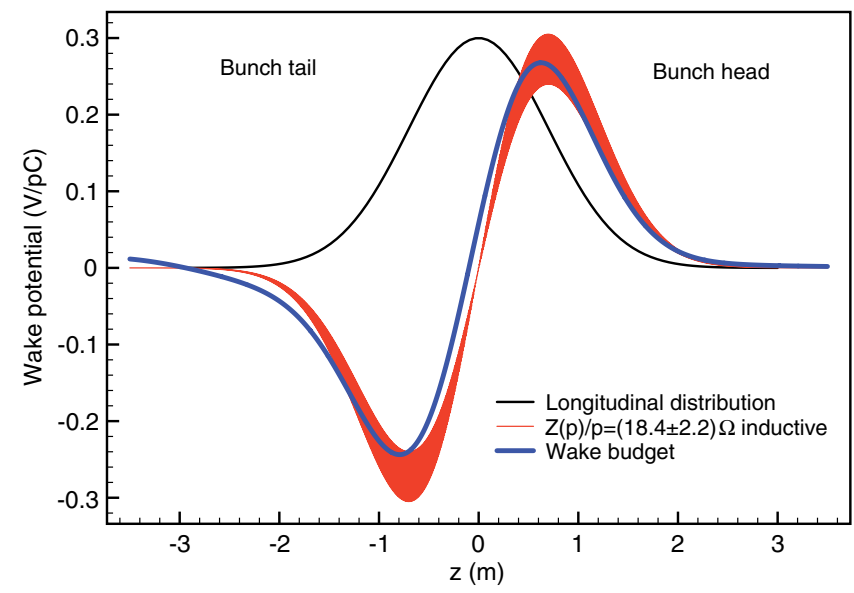

FIG. 16. Wake potential of a 2.3 ns Gaussian bunch given by the total impedance budget and by an inductive impedance model.

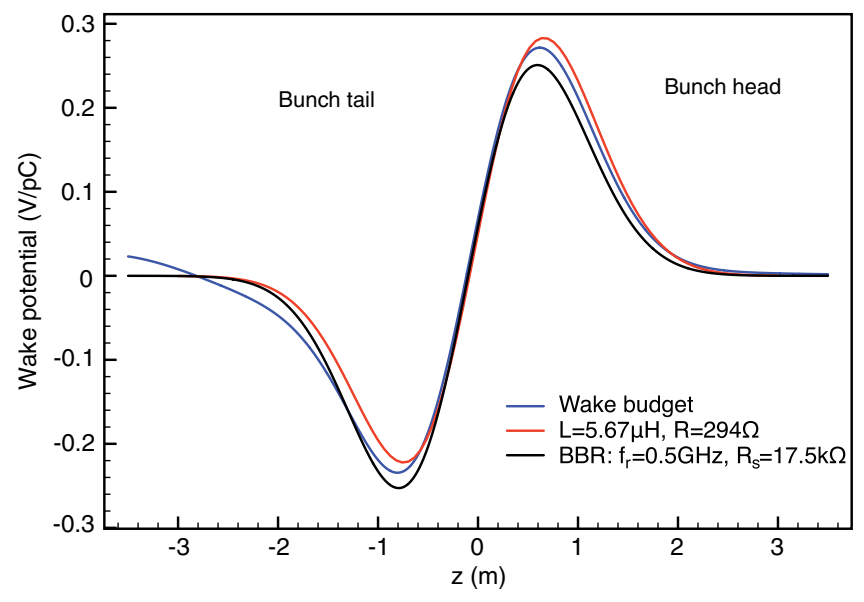

FIG. 17. Wake potentials of a 2.3 ns Gaussian bunch given by the Heifets-Bane model $Z(\omega)=\left(i 5.67 \times 10^{-6} \omega+294\right) \Omega$ and a broadband resonator with $f_{r}=0.5 \mathrm{GHz}$ and $R_{s}=17.5 \mathrm{k} \Omega$ compared to the total wake budget.

bunch of $2.3 \mathrm{~ns}$ is exactly the same of that of a pure inductive impedance.

An improved model of the machine impedance can be obtained by observing that there is a small asymmetry in the wake potential given by the impedance budget, which is mainly due to the resistive contribution to the impedance of the rf cavities and the ferrite loaded kickers. More precisely the impedance can then be derived from the Heifets-Bane model $[42,43]$, of which we maintain only the first two terms, the inductive and the resistive one, which best describe our particular impedance-generating elements. In Fig. 17, we show, with the red curve, the wake potential given by this improved impedance model, with an inductance of $L=5.67 \mu \mathrm{H}$, which corresponds to $Z(p) / p=17 i \Omega$, and a resistance of $R=294 \Omega$, which corresponds to $Z(p) / p=2 \Omega$ if evaluated at the bunch spectrum cutoff. Finally we observe that the wake potential can also be well fitted by a broadband resonator with a resonant frequency of $f_{r}=0.5 \mathrm{GHz}$, and a shunt impedance of $R_{s}=17.5 \mathrm{k} \Omega$, as shown with the black curve in the same figure. It is interesting to observe that this resonant frequency corresponds approximately to the frequency at which the real part of the kickers' longitudinal impedance has its maximum.

\section{CONCLUSIONS AND OUTLOOK}

The inductive broadband longitudinal coupling impedance of the CERN Proton Synchrotron has been measured from the incoherent quadrupole frequency shift as a function of single-bunch intensity, and the resulting value was $\operatorname{Im}[Z(p)] / p=(18.4 \pm 2.2) \Omega$. The procedure and the diagnostics now available can be used to monitor the impedance over the coming years, especially after new elements are added or others removed from the machine, also with a view to the LHC Injectors Upgrade project. 
Moreover, an analysis of the contributions to the total broadband impedance of several installations, such as ferrite loaded kickers, rf cavities, resistive wall, space charge, and many geometrical impedances, has been performed. The calculated total impedance at the frequency of the bunch spectrum cutoff is in good agreement with the measured effective impedance of the PS, assuming a parabolic distribution for the evaluation of the latter. We remember, however, that the numerical calculations with CST-Studio and ABCI are based on a Gaussian bunch. The inductive impedance model, or the improved models presented that also take into account a small resistive contribution to the total impedance, can be used to study, with the help of a simulation code, the beam dynamics under the effects of wakefields.

\section{ACKNOWLEDGMENTS}

We acknowledge useful discussions with G. Arduini, N. Biancacci, E. Métral, G. Rumolo, B. Salvant, E. Shaposhnikova, and V. Vaccaro. One of us, M. M., is grateful to the BE/ABP group for the warm hospitality during his stay at CERN. We also thank the PS operations team for the help in setting up the beams.

\section{APPENDIX}

In Table II we show the measured incoherent quadrupole frequency shift and bunch length (standard deviation $\sigma_{G}$ for Gaussian fit, total length $\tau_{b}$ for parabolic line density fit) at different beam intensities and rf peak voltages.

TABLE II. Measured incoherent quadrupole frequency shift and bunch length (standard deviation $\sigma_{G}$ for Gaussian fit, total length $\tau_{b}$ for parabolic line density fit) at different beam intensities and $\mathrm{rf}$ peak voltages.

\begin{tabular}{|c|c|c|c|c|}
\hline \multicolumn{5}{|c|}{ First set of measurements } \\
\hline$N_{p}\left(10^{11}\right)$ & $V_{\mathrm{RF}}(\mathrm{kV}) \pm 5 \%$ & $f_{2 s} \pm 12(\mathrm{~Hz})$ & $\sigma_{G}(\mathrm{~ns})$ & $\tau_{b}(\mathrm{~ns})$ \\
\hline $1.40 \pm 0.03$ & 80 & 960 & $2.35 \pm 0.01$ & $9.07 \pm 0.04$ \\
\hline $1.41 \pm 0.02$ & 40 & 675 & $2.83 \pm 0.02$ & $10.90 \pm 0.08$ \\
\hline $4.42 \pm 0.07$ & 40 & 620 & $3.65 \pm 0.03$ & $13.83 \pm 0.12$ \\
\hline $4.44 \pm 0.08$ & 80 & 895 & $2.95 \pm 0.02$ & $11.19 \pm 0.06$ \\
\hline $3.19 \pm 0.03$ & 80 & 915 & $2.54 \pm 0.01$ & $9.73 \pm 0.04$ \\
\hline $3.20 \pm 0.03$ & 40 & 640 & $3.12 \pm 0.01$ & $11.92 \pm 0.03$ \\
\hline $2.29 \pm 0.04$ & 40 & 640 & $2.83 \pm 0.02$ & $10.90 \pm 0.10$ \\
\hline $2.37 \pm 0.03$ & 80 & 930 & $2.39 \pm 0.03$ & $9.25 \pm 0.12$ \\
\hline $2.30 \pm 0.05$ & 80 & 930 & $2.33 \pm 0.03$ & $9.01 \pm 0.09$ \\
\hline $1.40 \pm 0.01$ & 80 & 955 & $2.94 \pm 0.02$ & $11.19 \pm 0.09$ \\
\hline $1.43 \pm 0.03$ & 40 & 690 & $3.55 \pm 0.02$ & $13.50 \pm 0.07$ \\
\hline \multicolumn{5}{|c|}{ Second set of measurements } \\
\hline$N_{p}\left(10^{11}\right)$ & $V_{\mathrm{RF}}(\mathrm{kV}) \pm 5 \%$ & $f_{2 s} \pm 12(\mathrm{~Hz})$ & $\sigma_{G}(\mathrm{~ns})$ & $\tau_{b}(\mathrm{~ns})$ \\
\hline $4.34 \pm 0.09$ & 47.5 & 690 & $3.53 \pm 0.03$ & $13.44 \pm 0.10$ \\
\hline $4.52 \pm 0.13$ & 95 & 980 & $2.92 \pm 0.02$ & $11.08 \pm 0.08$ \\
\hline $4.57 \pm 0.06$ & 47.5 & 710 & $3.56 \pm 0.005$ & $13.52 \pm 0.02$ \\
\hline $4.40 \pm 0.09$ & 95 & 970 & $2.90 \pm 0.007$ & $11.01 \pm 0.02$ \\
\hline $2.70 \pm 0.08$ & 47.5 & 720 & $3.30 \pm 0.01$ & $12.76 \pm 0.04$ \\
\hline $2.67 \pm 0.08$ & 95 & 1025 & $2.71 \pm 0.009$ & $10.48 \pm 0.03$ \\
\hline $2.69 \pm 0.04$ & 47.5 & 700 & $2.95 \pm 0.01$ & $11.35 \pm 0.05$ \\
\hline $2.62 \pm 0.08$ & 95 & 1020 & $2.42 \pm 0.02$ & $9.35 \pm 0.06$ \\
\hline $1.70 \pm 0.03$ & 47.5 & 760 & $3.45 \pm 0.007$ & $13.26 \pm 0.03$ \\
\hline $1.79 \pm 0.04$ & 95 & 1045 & $2.86 \pm 0.009$ & $11.06 \pm 0.03$ \\
\hline $1.76 \pm 0.03$ & 47.5 & 735 & $3.01 \pm 0.02$ & $11.59 \pm 0.05$ \\
\hline $1.79 \pm 0.03$ & 95 & 1025 & $2.49 \pm 0.01$ & $9.59 \pm 0.05$ \\
\hline $0.88 \pm 0.03$ & 47.5 & 765 & $3.27 \pm 0.01$ & $12.46 \pm 0.04$ \\
\hline $0.91 \pm 0.02$ & 95 & 1045 & $2.73 \pm 0.008$ & $10.41 \pm 0.03$ \\
\hline $0.90 \pm 0.04$ & 47.5 & 745 & $2.39 \pm 0.03$ & $9.32 \pm 0.10$ \\
\hline $0.90 \pm 0.02$ & 95 & 1040 & $1.97 \pm 0.02$ & $7.71 \pm 0.09$ \\
\hline
\end{tabular}


[1] S. Gilardoni et al., Proceedings of IPAC11, San Sebastián, Spain (EPS-AG, Spain, 2011), p. 2520.

[2] D. Boussard et al., Proceedings of PAC79, San Francisco, California (IEEE Trans. Nuclear Science, 1979), Vol. NS26, p. 3568.

[3] D. Boussard et al., CERN Report No. PS/OP/LR/Note 8016, CERN, Geneva, Switzerland, 1980.

[4] The quantity $Z(p) / p$ is the impedance evaluated at a frequency $p \omega_{0}$ with $p$ integer and $\omega_{0}$ the revolution angular frequency. It is often called in literature $Z / n$.

[5] S. Hancock (private communication).

[6] E. Shaposhnikova et al., Proceedings of the 23rd Particle Accelerator Conference, Vancouver, Canada, 2009 (IEEE, Piscataway, NJ, 2009), p. 4667.

[7] LHC beam extraction energy in the PS.

[8] D. Boussard and J. Gareyte, CERN SPS Improvement Report No. 181, CERN, Geneva, Switzerland, 1980.

[9] E. N. Shaposhnikova, CERN Report No. SL/94-19 (RF), CERN, Geneva, Switzerland, 1994.

[10] S. Y. Lee, Accelerator Physics (World Scientific, Singapore, 2004), 2nd ed., p. 261.

[11] D. Boussard, SPS Improvement Reports No. 167 and No. SPS/AC/JG/jf, 1979.

[12] J. L. Laclare, CERN Report No. 87-03, CERN, Geneva, Switzerland, 1987, p. 264.

[13] H. Damerau et al., CERN Report No. CERN-ATS-Note2012-064 MD, CERN, Geneva, Switzerland, 2012.

[14] R. Cappi et al., "Measurements of the PS low-frequency inductive broad-band impedance," PS Performance Committee meeting, December 2001, http://psdoc.web .cern.ch/PSdoc/ppc/ppc011214/emetral.pdf.

[15] D. Boussard, CERN Report No. CERN LABII/RF/Int./ 75-2, CERN, Geneva, Switzerland 1975.

[16] T. Bohl (private communication).

[17] R. Boni, A. Drago, A. Gallo, A. Ghigo, F. Marcellini, M. Migliorati, F. Sannibale, M. Serio, A. Stella, G. Vignola, and M. Zobov, Nucl. Instrum. Methods Phys. Res., Sect. A 418, 241 (1998).

[18] D. Alesini, A. Gallo, S. Guiducci, F. Marcellini, M. Zobov, M. Migliorati, and L. Palumbo, Phys. Rev. ST Accel. Beams 6, 074401 (2003).

[19] A. W. Chao, Physics of Collective Beam Instabilities in High Energy Accelerators (John Wiley \& Sons, New York, 1993).

[20] S. S. Kurennoy, AIP Conf. Proc. 496, 361 (1999).
[21] J. G. Wang, AIP Conf. Proc. 496, 276 (1999).

[22] R.L. Gluckstern, CERN Report No. CERN 2000-011, CERN, Geneva, Switzerland, 2000.

[23] L. Palumbo, V. G. Vaccaro, G. Wustefeld, IEEE Trans. Nucl. Sci. NS-31, 4 (1984).

[24] PS Multi-Turn Extraction Study Group, CERN Report No. CERN-2006-011, CERN, Geneva, Switzerland 2006.

[25] H. Tsutsui, Proceedings of the European Particle Accelerator Conference, Vienna, 2000 (EPS, Geneva, 2000), p. 1444.

[26] E. Métral et al., CERN Report No. CERN-AB-2006-051, CERN, Geneva, Switzerland, 2006.

[27] CST AG, Darmstadt, Germany, www.cst.com.

[28] C. Zannini et al., CERN Report No. CERN-BE-Note2010-006, CERN, Geneva, Switzerland, 2009.

[29] L. Palumbo and V. G. Vaccaro, Il Nuovo Cimento A 89, 3 (1985).

[30] R. L. Gluckstern, J. van Zeijts, and B. Zotter, Phys. Rev. E 47, 656 (1993).

[31] K. Yokoya, Part. Accel. 41, 221 (1993).

[32] P. Chiggiato (private communication).

[33] S. Y. Zhang, BNL/SNS Technical note No. 61, BNL, New York, 1999.

[34] M. Zobov et al., LNF Report No. LNF-95/041 (P), Frascati, Italy, 1995.

[35] Y.H. Chin, KEK Report No. 2005-06, KEK, Tsukuba, Japan, 2005.

[36] R. Losito, CERN Report No. PS/RF/Note 96-14, CERN, Geneva, Switzerland, 1996.

[37] J. B. García, and S. Gilardoni, Phys. Rev. ST Accel. Beams, 14, 030101 (2011).

[38] S. Kurennoy and G. Stupakov, Part. Accel. 45, 95 (1994).

[39] D. Davino, G. Dôme, G. Miano, G. Panariello, V. G. Vaccaro, and L. Verolino, Il Nuovo Cimento A 112, 12 (1999).

[40] S. A. Kheifets and S. A. Heifets, SLAC Report No. SLACPUB 3965, SLAC, Menlo Park, California, 1986.

[41] A. Hofmann and J. R. Maidment, CERN LEP note 168, CERN, Geneva, Switzerland, 1979.

[42] L. Palumbo, V. G. Vaccaro, and M. Zobov, CERN Report No. CERN 95-06, CERN, Geneva, Switzerland, 1995, pp. 331-390.

[43] S. Heifets, SLAC Report No. SLAC/AP-93, SLAC, Menlo Park, California, 1992. 